\title{
Weak Coulomb blockade effect in quantum dots
}

\author{
Piet W. Brouwer, ${ }^{1}$ Austen Lamacraft, ${ }^{2}$ and Karsten Flensberg ${ }^{3}$ \\ ${ }^{1}$ Laboratory of Atomic and Solid State Physics, Cornell University, Ithaca, New York 14853-2501 \\ ${ }^{2}$ Department of Physics, Princeton University, Princeton, NJ 08544, USA \\ ${ }^{3}$ Nano-Science Center, Niels Bohr Institute, Universitetsparken 5, 2100 Copenhagen, Denmark
}

(Dated: November 11, 2018)

\begin{abstract}
We develop the general non-equilibrium theory of transport through a quantum dot, including Coulomb Blockade effects via a $1 / N$ expansion, where $N$ is the number of scattering channels. At lowest order we recover the Landauer formula for the current plus a self-consistent equation for the dot potential. We obtain the leading corrections and compare with earlier approaches. Finally, we show that to leading and next leading order in $1 / N$ there is no interaction correction to the weak localization, in contrast to previous theories, but consistent with experiments by Huibers et al. [Phys. Rev. Lett. 81, 1917 (1998)], where $N=4$.
\end{abstract}

PACS numbers: 73.23.-b,73.21.La,73.23.Hk

The Landauer formula relates the two-terminal conductance of a mesoscopic conductor to its quantum mechanical scattering matrix 1]. This formula, and its extensions to multiterminal geometries and other transport properties, has become one of the cornerstones of the theory of quantum transport. However, because the Landauer formula relies on the single-particle scattering matrix, effects of electron-electron interaction are neglected.

In a chaotic quantum dot the dominant part of the interaction is 2,3$]$

$$
H_{c}=E_{c}\left(\hat{N}_{\mathrm{d}}-\mathcal{N}\right)^{2},
$$

where $E_{c}=e^{2} / 2 C$ is the one-electron charging energy in terms of the dot's capacitance $C, \hat{N}_{\mathrm{d}}$ is the number of electrons on the quantum dot, and $\mathcal{N}$ is an offset determined by the voltages on nearby gates. The simplest way to include this interaction into the scattering formalism is to replace $H_{c}$ by a self-consistent potential

$$
V_{\mathrm{d}}(t)=-\frac{e}{C}\left(\left\langle\hat{N}_{\mathrm{d}}(t)\right\rangle-\mathcal{N}\right) .
$$

where the expectation value of the number of electrons $\left\langle\hat{N}_{\mathrm{d}}\right\rangle$ is computed in the presence of the potential $V_{\mathrm{d}}$. The self-consistent potential is of particular interest for nonlinear and time-dependent transport [4]. It is generally believed that Eq. (2) is valid when the charge on the dot fluctuates freely, either due to large contact conductance or high temperatures, in which cases Coulomb blockade is suppressed [3]. However, in this limit no systematic evaluation of the corrections to the quantum transport has been given for general non-equilibrium conditions. In this Letter, we develop a theory of non-equilibrium transport that reproduces the Landauer formula with the self-consistent potential at lowest order and admits corrections as a power series in the inverse of the number of scattering channels $N$.

The situation we study is thus referred to as 'weak Coulomb blockade' [3], as a hard Coulomb gap in the current-voltage characteristic is not present. Historically, the influence of quantum charge fluctuations on the

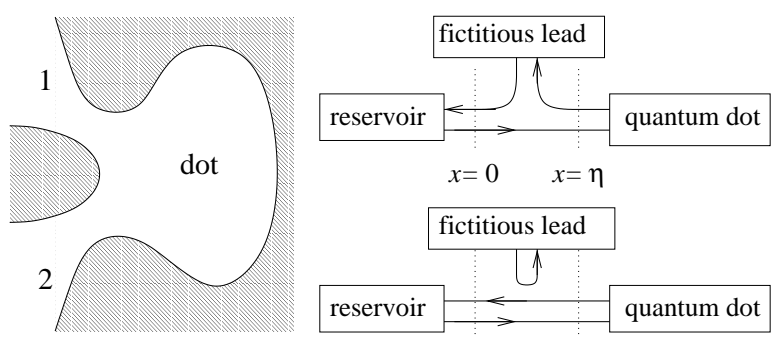

FIG. 1: Left: Schematic drawing of a quantum dot coupled to source and drain leads via point contacts. Right, top: a fictitious lead is side-coupled to electrons leaving the dot as a mathematical trick. The scattering Hamiltonian $H_{1}$, when included to all orders, decouples the fictitious leads, as illustrated in the bottom right panel.

transport properties of mesoscopic junctions first focused on the coupling to the 'electromagnetic environment' of an electron moving through a single junction [5]. This situation was recently extended to a single open contact in large- $N$ limit, similar in spirit to the present paper, but restricted to a point scatterer [6]. To deal with the strong quantum charge fluctuations of almost fully transmitting point contacts, Flensberg 7] and Matveev 8] introduced a bosonized description (see also Ref. 9). The weak Coulomb blockade situation has also been addressed starting from the tunnel junction limit, using the phase as variable [10], and good agreement with experiments on large conductance metallic systems has been achieved [11]. Recently, the technique was extended to junctions of arbitrary transparency 12]. A crucial simplification of all these approaches was that once an electron enters the dot, it never coherently returns to the leads. This was assumed to be satisfactory for large dots where the typical dwell time $\tau_{\mathrm{d}}$ by far exceeds the time scale set by inelastic processes.

For the problem we consider here, the coherent return of the electrons to the point contacts is crucial. Only then is transport in the absence of interactions described 
by a unitary scattering matrix $S$. For the coherent case, the interaction correction to the dc conductance of an open quantum dot was first calculated in the bosonization [3, 13 and later in the phase representation formalisms [14]. The results of these two approaches are strikingly different. Our secondary aim in this Letter is to clarify this situation. In this regard, we find that the result of Refs. 3, 13], which is a formal expansion in the scattering matrix $S$, is not applicable for unitary $S$, despite claims to the contrary. However, it is applicable for a subunitary scattering matrix, which may serve as a phenomenological description of a quantum dot with strong relaxation. On the other hand, the $1 / N$ corrections we obtain here are fully consistent with the analysis of Ref. 14]. Furthermore, whereas Ref. 14] intended to describe the case of metallic quantum dots, i.e., neglecting quantum corrections to the non-interacting conductance, we find that the same expression for the conductance also describes the interaction correction to quantum interference corrections. Thus our theory applies to experiments on the weak localization corrections to conductance [15].

We now turn to a description of our calculation. We consider a quantum dot coupled to source and drain leads through point contacts with $N_{1}$ and $N_{2}$ channels respectively $\left(N_{1}+N_{2}=N\right)$, see Fig. 11 The starting point of our calculation is the one-dimensional description of the electrons in the point contacts [7, 8]

$$
\begin{gathered}
H_{0}=i v_{F} \sum_{j=1}^{N} \int d x\left[\hat{\psi}_{L j}^{\dagger}(x) \frac{\partial}{\partial x} \hat{\psi}_{L j}(x)\right. \\
\left.-\hat{\psi}_{R j}^{\dagger}(x) \frac{\partial}{\partial x} \hat{\psi}_{R j}(x)\right]+H_{c} .
\end{gathered}
$$

Here $v_{F}$ is the Fermi velocity (differing velocities in each channel may be removed by rescaling), $\psi_{L j}(x)$ and $\psi_{R j}$ describe left and right moving fermions in channel $j, H_{c}$ is given by Eq. (1), and we set $\hbar=1$. The lead-dot interface is taken at $x=0$, the region $x<0$ corresponding to the leads. Following Ref. 16, we write the number of electrons in the dot as

$$
\hat{N}_{\mathrm{d}}=N_{0}-\int_{-\infty}^{0} d x:\left[\hat{\psi}_{L j}^{\dagger}(x) \hat{\psi}_{L j}(x)+\hat{\psi}_{R j}^{\dagger}(x) \hat{\psi}_{R j}(x)\right]:
$$

where $N_{0}$, the combined number of electrons in the dot and the leads, is taken to be a constant.

The fields $\psi_{L}$ and $\psi_{R}$ in Eq. (3) are not independent, since they are connected by the dot. After the substitution (4), which removes interactions inside the dot, their relationship is described by the scattering matrix

$$
\hat{\psi}_{L i}(\eta, t)=\sum_{j} \int d t^{\prime} S_{i j}\left(t, t^{\prime}\right) \psi_{R j}\left(\eta, t^{\prime}\right),
$$

where $\eta$ is a positive infinitesimal. (Scattering from a quantum dot with time-dependent potentials is described by a scattering matrix with two time arguments, see e.g., Ref. [17.)
Instead of solving the interacting Hamiltonian (3) with the boundary condition (5), we reformulate the problem using a trick that allows a treatment with standard perturbative techniques. Our method is similar in spirit to that of Refs. 3, 13, 16]. As shown in Fig. 1, we couple a fictitious reservoir to the left-moving electrons in the point contact. Then the problem reduces to the exactly solvable theory of Flensberg and Matveev, where electrons that enter the quantum dot never return coherently. We restore the original model by adding a term

$$
H_{1}=2 v_{F} \sum_{j=1}^{N}\left(\hat{\psi}_{L j}^{\dagger}(0) \hat{\psi}_{L j}(\eta)+\hat{\psi}_{L j}^{\dagger}(\eta) \hat{\psi}_{L j}(0)\right),
$$

to the one-dimensional Hamiltonian Eq. (3), thus shunting the fictitious leads and regaining coherent scattering by the quantum dot.

We proceed by evaluating the current in perturbation theory in $H_{1}$ using the Keldysh formalism and Eq. (5),

$$
I(t)=\left\langle T_{\mathrm{c}} \hat{I}(t) e^{-i \int_{\mathrm{c}} d t^{\prime} \hat{H}_{1}\left(t^{\prime}\right)}\right\rangle_{0} .
$$

Here $T_{\mathrm{c}}$ denotes ordering along the Keldysh contour c and the average is taken with respect to the Hamiltonian $H_{0}$. The current $I$ is calculated as the weighted difference of the currents in the two point contacts,

$$
\hat{I}=-e v_{F} \sum_{j} \Lambda_{j j}\left[\hat{\psi}_{R j}^{\dagger}(0) \hat{\psi}_{R j}(0)-\hat{\psi}_{L j}^{\dagger}(0) \hat{\psi}_{L j}(0)\right],
$$

where $\Lambda_{i j}=\delta_{i j}\left(N_{2} / N\right)$ for $j=1, \ldots, N_{1}$ and $\Lambda_{i j}=$ $-\delta_{i j}\left(N_{1} / N\right)$ for $j=N_{1}+1, \ldots, N$.

For the perturbative evaluation of Eq. (7) we require the correlation function [3]

$$
\begin{aligned}
& \left\langle T_{c} \prod_{p, q} \hat{\psi}_{L i_{p}}^{\dagger}\left(t_{p}\right) \hat{\psi}_{L l_{p}}\left(s_{p}\right) \hat{\psi}_{R j_{q}}\left(t_{q}^{\prime}\right) \hat{\psi}_{R k_{q}}^{\dagger}\left(s_{q}^{\prime}\right)\right\rangle_{0}= \\
& \langle\cdots\rangle_{\mathrm{ni}} \times \prod_{p, q}\left(\frac{f\left(t_{p}, t_{q}^{\prime}\right) f\left(s_{p}, s_{q}^{\prime}\right)}{f\left(t_{p}, s_{q}^{\prime}\right) f\left(s_{p}, t_{q}^{\prime}\right)}\right)^{1 / N},
\end{aligned}
$$

(here all fields are taken at $x=0$ ) where $\langle\cdots\rangle_{\text {ni }}$ represents the corresponding correlation function of the noninteracting theory (with $E_{c} \rightarrow 0$ ) and

$$
\begin{aligned}
\ln f\left(t, t^{\prime}\right)= & \text { const. }-\frac{E_{c} N}{\pi} \int_{0}^{\infty} d \zeta e^{-E_{c} N \zeta / \pi} \\
& \times \ln \frac{\sinh \left[\pi T\left(t-t^{\prime}+\zeta-i 0^{+} \operatorname{sgn}_{c}(t)\right)\right]}{\sinh [\pi T \zeta]} .
\end{aligned}
$$

Here $\operatorname{sgn}_{c}(t)$ is \pm 1 for the forward and backward branches of the Keldysh contour respectively. Additional 'anomalous' correlators containing $N \hat{\psi}_{L}$ and $N \hat{\psi}_{R}^{\dagger}$ (or vice versa) give oscillating contributions to the current. These appear at $N^{\text {th }}$ order in perturbation theory, so they are not captured by $1 / N$ methods [3]. 
Expanding Eq. (17) to second order in $H_{1}$ is now a straightforward calculation. The result is

$$
\begin{aligned}
I(t)= & -\frac{e}{2 \pi} \operatorname{tr} \Lambda \mu_{R}-\frac{i e v_{F}}{2} \int d t^{\prime} d s^{\prime} \operatorname{tr}\left[\Lambda S\left(t, t^{\prime}\right) G_{R}^{K}\left(t^{\prime}, s^{\prime}\right) S^{\dagger}\left(s^{\prime}, t\right)\right] \\
& -e v_{F}^{2} \operatorname{Im} \int d s d t^{\prime} d s^{\prime} \operatorname{tr}\left[\Lambda G_{L}^{K}(s, t) S\left(t, t^{\prime}\right) G_{R}^{K}\left(t^{\prime}, s^{\prime}\right) S^{\dagger}\left(s^{\prime}, s\right)\right] \sin \left[\kappa_{0}\left(s, t^{\prime}\right)\right]\left|\frac{f\left(t, t^{\prime}\right) f\left(s, s^{\prime}\right)}{f\left(t, s^{\prime}\right) f\left(s, t^{\prime}\right)}\right|^{1 / N},
\end{aligned}
$$

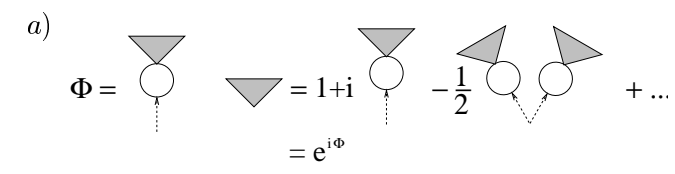

b)
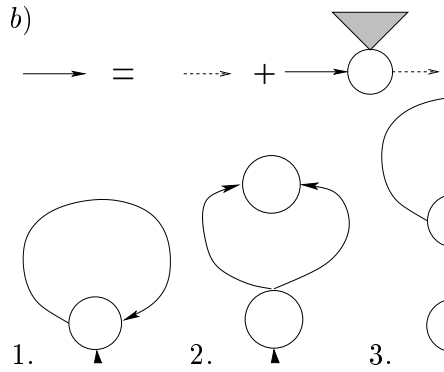

2
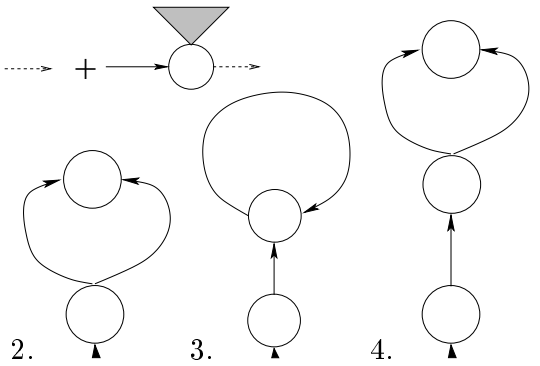

FIG. 2: a) At leading order in $1 / N$, we retain only tree diagrams. b) The one-loop diagrams

where we wrote $N \kappa_{0}\left(t, t^{\prime}\right) \operatorname{sgn}_{\mathrm{c}}(t)=\operatorname{Im} \ln f\left(t, t^{\prime}\right)$, i.e.,

$$
\kappa_{0}\left(t, t^{\prime}\right)=\frac{\pi}{N}\left(1-e^{-E_{c} N\left(t^{\prime}-t\right) / \pi}\right) \theta\left(t^{\prime}-t\right) .
$$

In Eq. (11) the trace is over the scattering channels $j=$ $1, \ldots, N$. The Keldysh Green's function $G_{R i j}^{K}(t, s)$ is

$$
G_{R i j}^{K}(t, s)=-\frac{T}{v_{F}} \delta_{i j} \mathrm{P} \frac{e^{-i \int_{s}^{t} d \sigma \mu_{R i}(\sigma)}}{\sinh [\pi T(t-s)]},
$$

where "P" denotes the Cauchy principal value and $\mu_{R i}$ is the (electro-)chemical potential of electrons entering the dot through through the $i$ th channel, $i=1, \ldots, N$. With zero chemical potential for the left movers, electroneutrality demands $\sum_{j} \mu_{R j}=0$. A similar expression holds for $G_{L}^{\mathrm{K}}$, but without the phase factor in the exponent.

The linear dc conductance of Refs. 3, 13 is recovered upon taking $\mu_{R i}=-e V_{\mathrm{sd}} \Lambda_{i i}, i=1, \ldots, N$, setting $S\left(t, t^{\prime}\right)=S\left(t-t^{\prime}\right)$, and expanding Eq. (11) in the sourcedrain voltage $V_{\mathrm{sd}}$. Truncating the perturbation theory in $H_{1}$ at second order corresponds to a formal expansion up to second order in the scattering matrix $S-$ which is precisely what was done in Refs. 13,13 . However, as $S$ is unitary, we find no justification for stopping here. That this approach gives unphysical results may be readily seen by substituting into Eq. (11) the scattering matrix of a ballistic quantum wire with no backscattering, resulting in a spurious correction to the Landauer conductance of $e^{2} / h$ per spin channel.
In order to do a calculation to all orders in $H_{1}$, we need a different principle to organize the perturbation theory. In fact, the correlation function (9) shows that a systematic expansion in $1 / N$ is possible [keeping $E_{C} N$ in Eq. (12) constant], while including $H_{1}$ to all orders. The result is a sum over partitions into 'bubbles' in standard diagrammatic language. Each bubble is formally of order $N$, as it involves a trace over the channel index. In order to be non-vanishing, bubbles must be connected by 'interaction lines' $N^{-1} \ln f\left(t, t^{\prime}\right)$. Denoting these by dashed arrows, as in Fig. $2 \mathrm{k}$, the arrowhead to indicate the unprimed time, we see that at leading order we must have precisely one factor $N^{-1} \ln f\left(t, t^{\prime}\right)$ for every bubble, except for the bubble that contains the current operator. The result is a tree structure, as shown in Fig. 212. We thus find

$$
\begin{aligned}
I(t)= & -\frac{e}{2 \pi} \operatorname{tr} \Lambda \mu_{R} \\
& -\frac{i e v_{F}}{2} \int d t^{\prime} d s^{\prime} \operatorname{tr} \Lambda S\left(t, t^{\prime}\right) \tilde{G}_{R}^{K}\left(t^{\prime}, s^{\prime}\right) S^{\dagger}\left(s^{\prime}, t\right) .
\end{aligned}
$$

Here $\tilde{G}_{R}^{K}\left(t^{\prime}, s^{\prime}\right)=G_{R}^{K}\left(t^{\prime}, s^{\prime}\right) e^{-i \tilde{\phi}\left(t^{\prime}, s^{\prime}\right)}$ where $\tilde{\phi}\left(t_{1}^{\prime}, t_{2}^{\prime}\right)$ satisfies the self-consistent equation (see Fig. [2]),

$$
\begin{aligned}
\tilde{\phi}\left(t^{\prime}, s^{\prime}\right) & =2 \int_{-\infty}^{\infty} d t j_{c}(t)\left[\kappa_{0}\left(t, t^{\prime}\right)-\kappa_{0}\left(t, s^{\prime}\right)\right], \\
j_{c}(t) & =\frac{i v_{F}}{2} \operatorname{tr}\left[G_{L}^{\mathrm{K}}(t, t)\right. \\
& \left.-\int d t_{1}^{\prime} d s_{1}^{\prime} S\left(t, t_{1}^{\prime}\right) \tilde{G}_{R}^{K}\left(t_{1}^{\prime}, s_{1}^{\prime}\right) S^{\dagger}\left(s_{1}^{\prime}, t\right)\right] .
\end{aligned}
$$

The current $j_{c}$ is the particle current leaving the dot due to scattering inside. The quantity $\tilde{\phi}\left(t^{\prime}, s^{\prime}\right)$ may be thought of as the difference in phases accumulated by electrons that enter the dot at times $t^{\prime}$ and $s^{\prime}$ in the presence of a Coulomb potential. Indeed, writing $\tilde{\phi}\left(t^{\prime}, s^{\prime}\right)=\int_{s^{\prime}}^{t^{\prime}} d t e V_{\mathrm{d}}(t)$ and using Eq. (12) for $\kappa_{0}$, the self-consistency relation (15) takes the simple form

$$
C \partial_{t} V_{\mathrm{d}}(t)=e j_{c}(t)-\left(N e^{2} / 2 \pi\right) V_{\mathrm{d}}(t),
$$

which is precisely the equation for the dot potential in the self-consistent treatment of the interaction Hamiltonian (11). Hence, evaluating the current at tree level, we recover precisely the Landauer formula Eq. (14), including the approximation (22) for the interaction [4]. The leading correction to the above self-consistent theory adds one more 'interaction line' to each tree, which 
results in diagrams with one loop, and generates the RPA-like series in Fig. 2p. The summation of these leads to a renormalization of the interaction line Eq. (12): $\kappa\left(t, t^{\prime}\right)=\kappa_{0}\left(t, t^{\prime}\right)+\int d t_{1} \chi\left(t, t_{1}\right) \kappa\left(t_{1}, t^{\prime}\right)$, where

$$
\chi\left(t, t_{1}\right)=2 \int d t_{1}^{\prime} d s_{1}^{\prime} \frac{\delta j_{c}\left(t_{1}\right)}{\delta \tilde{\phi}\left(t_{1}^{\prime}, s_{1}^{\prime}\right)}\left[\kappa_{0}\left(t, t_{1}^{\prime}\right)-\kappa_{0}\left(t, s_{1}^{\prime}\right)\right] .
$$

The four one-loop diagrams are shown in Fig. 20, where the solid interaction lines denote $\kappa\left(t, t^{\prime}\right)[19]$. We thus find the leading interaction correction to the current is

$$
I_{1}(t)=e v_{F}^{2} \operatorname{Im} \int d t_{1} d t_{1}^{\prime} d s_{1}^{\prime} d t_{2}^{\prime} d s_{2}^{\prime} \kappa\left(t_{1}, t_{2}^{\prime}\right) \operatorname{tr} S\left(t_{1}, t_{1}^{\prime}\right) \tilde{G}_{R}^{K}\left(t_{1}^{\prime}, s_{1}^{\prime}\right) S^{\dagger}\left(s_{1}^{\prime}, t\right) \Lambda S\left(t, t_{2}^{\prime}\right) \tilde{G}_{R}^{K}\left(t_{2}^{\prime}, s_{2}^{\prime}\right) S^{\dagger}\left(s_{2}^{\prime}, t_{1}\right),
$$

leading to our final result for the one-loop correction to the dc conductance

$$
G_{1}=\frac{e^{2}}{8 \pi^{2} T} \operatorname{Im} \int d \varepsilon d \omega \frac{\tanh [(\varepsilon+\omega) / 2 T]}{\cosh ^{2}[\varepsilon / 2 T]} \kappa(\omega) \operatorname{tr}\left[\Lambda S(\varepsilon) \Lambda S^{\dagger}(\varepsilon+\omega)-\Lambda S(\varepsilon) S^{\dagger}(\varepsilon+\omega) S(\varepsilon) \Lambda S^{\dagger}(\varepsilon)\right] .
$$

The result (18) coincides with that found in Ref. [14]. We note that it gives not only the leading interaction correction to the semiclassical conductance, but also to the quantum interference corrections. In particular, an ensemble average of Eq. (18) predicts the absence of an interaction correction to weak localization, which is in disagreement with the conclusions of Ref. 13, but consistent with experiments by Huibers et al., who find that weak localization is well described by the non-interacting theory, suitably modified for the presence of a small amount of dephasing, down to the lowest temperatures available [15]. There is a simple reason for this. The interaction correction Eq. (18) derives from a renormalized scattering matrix $S^{\prime}(\varepsilon)=S(\varepsilon)+\delta S(\varepsilon)$, with $[14,18]$

$$
\begin{aligned}
\delta S(\varepsilon)= & -i \int \frac{d \omega}{2 \pi} \kappa(\omega)[\tanh [(\varepsilon-\omega) / 2 T] S(\varepsilon-\omega) \\
& \left.+\tanh [(\varepsilon+\omega) / 2 T] S(\varepsilon) S^{\dagger}(\varepsilon+\omega) S(\varepsilon)\right] .
\end{aligned}
$$

This correction preserves, at each energy, the circular invariance of the ensemble of scattering matrices that describes open dots. Quantities like the weak localization correction, that do not depend on correlations at different energies, are thus completely unaffected by the replacement $S(\varepsilon) \rightarrow S^{\prime}(\varepsilon)$. Note that this is a statment about the ensemble averaged conductance, and the conductance of any particualar dot configuration will acquire a temperature-dependent renormalization of random sign. We note that for non-ideal contacts with reflection matrices, $r_{\mathrm{c} j}, j=1,2$, Eq. (18) predicts the renormalization of the classical conductance $g \equiv g_{1} g_{2} /\left(g_{1}+g_{2}\right)$

$\delta g_{j}=-2 \operatorname{tr} r_{\mathrm{c} j} r_{\mathrm{c} j}^{\dagger}\left(1-r_{\mathrm{c} j} r_{\mathrm{c} j}^{\dagger}\right) \frac{1}{g} \ln \frac{E_{\mathrm{c}} g e^{1+\mathrm{C}}}{2 \pi^{2} T}, \tau_{\mathrm{d}}^{-1} \ll T \ll g E_{\mathrm{c}}$. $g_{j}=N_{j}-\operatorname{tr} r_{\mathrm{c} j} r_{\mathrm{c} j}^{\dagger}$ is the conductance of each contact $[\underline{6}]$.
We have presented a formalism that elucidates the relationship between the conflicting theories of weak Coulomb blockade [3, 13, 14]. Our formalism provides systematic corrections to the use of a self-consistent potential in the scattering approach to quantum transport.

We would like to thank Igor Aleiner and Leonid Glazman for discussion of the results. This work was supported by the NSF under grant no. DMR 0334499 and by the Packard Foundation and by the Danish Natural Science Reseach Council.

[1] See, e.g., Y. Imry, Introduction to Mesoscopic Physics (Oxford University Press, New York, 1997).

[2] I. L. Kurland, I. L. Aleiner, and B. L. Altshuler, Phys. Rev. B 62, 14886 (2002).

[3] I. L. Aleiner, P. W. Brouwer, and L. I. Glazman, Phys. Rep. 358, 309 (2002).

[4] M. Büttiker, A. Prêtre, and H. Thomas, Phys. Rev. Lett. 70, 4114 (1993).

[5] G.-L. Ingold and Y. V. Nazarov, in Single Charge Tunneling, edited by H. Grabert and M. H. Devoret (Plenum, New York, 1992), Chap. 2.

[6] D. S. Golubev and A. D. Zaikin, Phys. Rev. Lett. 86, 4887, (2001).

[7] K. Flensberg, Phys. Rev. B 48, 11156 (1993).

[8] K. A. Matveev, Phys. Rev. B 51, 1743 (1995).

[9] A. Furusaki and K. A. Matveev, Phys. Rev. B. 52, 16676 (1995).

[10] G. Falci, G. Schön, and G. T. Zimanyi, Phys. Rev. Lett. 74, 3257 (1995); X. Wang, R. Egger, and H. Grabert, Europhys. Lett. 38, 545 (1997); J. König and H. Schoeller, Phys. Rev. Lett. 81, 3511 (1998).

[11] D.S. Golubev, J. König, H. Schoeller, G. Schön, and A. D. Zaikin, Phys. Rev. B 56, 15782 (1997).

[12] Yu. V. Nazarov, Phys. Rev. Lett. 82, 1245, (1999).

[13] P. W. Brouwer, I. L. Aleiner, Phys. Rev. Lett. 82, 390 
(1999)

[14] D. S. Golubev and A. D. Zaikin, Phys. Rev. B 69, 075318 (2004).

[15] A. G. Huibers, S. R. Patel, C. M. Marcus, P. W. Brouwer, C. I. Duruöz, and J. S. Harris, Phys. Rev. Lett. 81, 1917 (1998).

[16] I. L. Aleiner and L. I. Glazman, Phys. Rev. B 57, 9608
(1998).

[17] M. L. Polianski and P. W. Brouwer, J. Phys. A 36, 3215 (2003).

[18] P. W. Brouwer, A. Lamacraft, and K. Flensberg, unpublished.

[19] Only the first diagram contributes to the dc conductance. 